\title{
Treatment in the home setting with intermittent pneumatic compression for a woman with chronic leg ulcers: a case report
}

\author{
Katrina Young ${ }^{1 *}$, Harrison Ng Chok ${ }^{2,3}$ and Lesley Wilkes ${ }^{4,3}$
}

\begin{abstract}
Background: Intermittent Pneumatic Compression (IPC) is shown to improve the healing rate of Venous Leg Ulcers $(V L U)$ in the hospital setting. The current Australian "Gold Standard" treatment according to the Australian and New Zealand Wound Management Associations' (AWMA) Prevention \& Management of Venous Leg Ulcer guidelines is compression, generally in the form of bandaging then progressing to hosiery once wounds are healed to prevent recurrence. This is recommended in conjunction with other standards of wound management including; nutrition, exercise, client education and addressing underlying pathophysiology and psychosocial factors. Compression bandaging is predominantly attended by community nurses in the clients' home. Barriers to delivery of this treatment include; client concordance and or suitability for bandaging including client habitus, (shape of legs), client lifestyle, clinician knowledge and clinicians physical ability to attend bandaging, in particular for obese clients with limited mobility who pose a manual handling risk to the clinician themselves. The use of IPC may assist in mitigating some of these concerns, therefore it would seem wise to explore the use of IPC within the home setting.
\end{abstract}

Case presentation: This paper will present an original case report on the successful treatment of a woman living with chronic bilateral lower leg ulcers using IPC as an adjunct treatment in her home. This paper supports recommendations to explore the use of IPC therapy in the home setting, for treatment of chronic leg ulcers requiring compression.

Conclusion: Use of IPC in the home is anticipated to improve client involvement, concordance, client outcomes and reduce risk to staff applying conventional compression bandaging systems, particularly for obese clients with limited mobility.

Keywords: Intermittent Pneumatic Compression (IPC), Chronic Leg Ulcer (CLU), Venous Leg Ulcer (VLU), Chronic Venous Insufficiency (CVI), Lymphoedema, Case report, Home setting

\section{Background}

The incidence of Australians living with a chronic leg ulcer (CLU) has not been accurately determined. It is estimated that up to $3 \%$ of the Australian population will develop a chronic venous leg ulcer in their lifetime [1], with McGuiness [2] reporting that approximately 400,000 people in Australia will suffer a CLU at any one time. Prevalence of chronic leg ulceration is rising in Australian society as a

\footnotetext{
*Correspondence: katrina.young@health.nsw.gov.au

${ }^{1}$ Nepean Hospital, Nepean Blue Mountains Local Health District, Derby Street, Kingswood, NSW 2747, Australia

Full list of author information is available at the end of the article
}

result of our ageing population and increasing risk factors such as diabetes, obesity, peripheral vascular disease, congestive cardiac failure, arthritis, injury and respiratory diseases [3]. CLU is defined as a defect in the skin below the knee that does not progress through the normal phases of healing and generally is considered chronic when it persists for longer than three months [4]. Chronic wounds are a poorly recognised chronic disease that causes pain and suffering and costs the Australian healthcare system an estimated $\mathrm{A} \$ 2.85$ billion dollars per annum $[5,6]$. The reported cost for acute management of CLUs alone was estimated at between $\mathrm{A} \$ 553$ and $\mathrm{A} \$ 654$ million in 2002 [7]. Not only 
does CLU pose a significant economic cost to the Australian healthcare system, but there is also a substantial personal cost [6]. Living with chronic wounds has a negative psychological impact on a person, often leading to loss of control, social isolation and depression [8]. To improve outcomes for individuals, they should be given the opportunity to understand their condition, be involved in their treatment, and have access to resources encouraging them to take responsibility for managing their wound where possible [9].

IPC is a device that uses an air pump to inflate and deflate an airtight garment placed around the legs, which mimics the rhythmic calf muscle contractures and promotes improved venous flow and lymphatic drainage [10]. IPC is also used as a treatment for deep vein thrombosis (DVT) prevention and it has been shown to improve healing of CLU $[11,12]$. A review of the literature supported further exploration of the use of IPC as an adjunct therapy for treatment of CLU of venous aetiology [12, 13]. IPC should be utilised with a holistic approach to wound management encompassing client related factors, wound related factors, environmental factors, skills and knowledge of clinicians, resource and treatment related factors [14]. Concordance with traditional forms of compression bandaging and hosiery is a challenge faced by healthcare professionals in the provision of compression therapy $[15,16]$.

Although compression using bandages or hosiery and calf pump exercises is proven to help healing and prevent recurrence of ulcers, many people do not adhere to compression therapy [12, 17]. There are a variety of reasons for non-concordance including pain, discomfort and inadequate lifestyle advice by healthcare providers, as reported by clients with leg ulcers $[18,19]$, additional reasons outlined by Van Hecke et al. [19] for non-concordance are difficulties in applying compression, skin problems, uncomfortable footwear, cosmetic appearance of bandages and financial restrictions prompting AWMA to push for a subsidised scheme for compression therapy in Australia as the high cost of therapy puts it out of reach for many Australians [2].

Whether or not IPC is better than compression bandaging has not been established, but the consensus is IPC is better for healing ulcers than no compression and improves outcomes for people with CLU $[12,17]$. This case report outlines one woman's journey living with CLU and treatment using IPC in her home, which will be presented using the CARE checklist [20].

\section{Timeline}

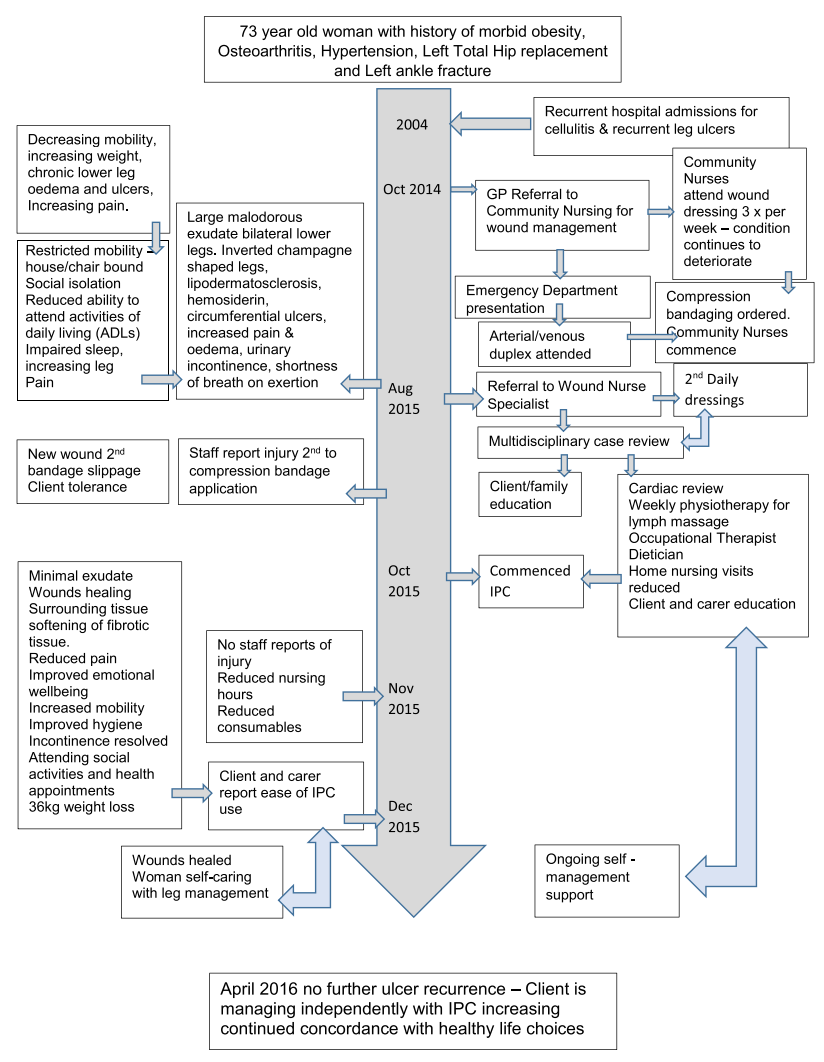




\section{Case presentation}

\section{Patient information}

An elderly woman was referred to Community Health in October 2014 for management of chronic lower leg ulcers (table 1). The woman had suffered recurrent hospital admissions throughout the prior ten years for lower leg cellulitis and leg ulcers, with three admissions within the previous two year period. A complex interplay of factors led to her ulcer development, including risk factors such as age and sex, relevant background history such as osteoarthritis, fractured left ankle, left total hip replacement, and morbid obesity leading to decreased mobility and calf pump action, causing chronic venous insufficiency of the superficial veins which also caused chronic lower leg oedema and lymphoedema. The woman reported the impact that the chronic ulcers were having on her life including; increasing pain, large amounts of odorous discharge, decreased mobility leading to increasing weight gain, lack of sleep, urinary incontinence, inability to shower, lack of independence, lack of energy, mood changes and social isolation.

Previous treatments had included antibiotic therapy and basic dressings with the General Practitioner (GP), hospital admissions for leg elevation and intravenous antibiotics. Access to specialised lymphoedema treatment was limited for two primary reasons. Firstly, she had become house bound with restricted transport to health specialists, and secondly, she lived in a geographically remote area. Community nurses commenced applying a two layer short stretch compression bandaging system in October 2014 as per previous hospital discharge instructions. The procedure required two community nurses to attend two to three times per week. A wound specialist review occurred in August 2015 following the woman's discharge from the emergency department where she had presented for review for increasing pain, redness and swelling of her legs. It had been noted that she had not been receiving consistent compression bandaging. The community nurses reported adverse events from bandaging the woman's legs including tissue damage from bandage slippage, friction and pressure and increased knee and thigh oedema with some nurses reporting personal injuries and difficulty with manual application of bandaging, the woman understood the need for compression but found it difficult to tolerate the bandaging due to pain at times.

\section{Physical exam}

\section{Diagnostic assessment}

The Wound Nurse Specialist examination in August 2015 noted significant oedema of the woman's lower legs, thighs. Bilateral lower legs had circumferential superficial ulcers with a copious amount of malodorous greenish exudate on the removed dressing, the presence of pseudomonas was confirmed by a wound swab. The skin of both legs was thickened with fibrosis, lipodermatosclerosis, venous eczema, and hemosiderin staining with hyperkeratosis and surrounding tissue maceration (Figs. 1 \& 2). The woman rated her wound and lower leg pain at an 8 on the Wound Assessment Tool pain scale (i.e. 0 equates to no pain to 10 extreme pain). Her right thigh circumference was $89 \mathrm{~cm}$ and Left thigh $93 \mathrm{~cm}$ and she had multiple skin folds with skin irritation because of poor hygiene and incontinence. She also had Papillomatosis wart like projections which were visualised on her inner thighs, her vital signs were stable and within acceptable limits whilst resting. Her height measured at $154 \mathrm{~cm}$ with a weight of $149 \mathrm{~kg}$ giving her a Body Mass Index (BMI) 62.83.

The woman's mobility was limited requiring assistance to stand from the sitting position, she mobilised with a four wheeled walker for five steps with wide stiff legged gait she was unable to attend knee bends or heel lifts, client became short of breath on exertion. Because of her reduced mobility she had been unable to sleep in bed remaining predominantly chair bound. The woman scored 20 on the Waterlow pressure injury risk assessment, placing her at very high risk for pressure ulcer development with a score of 10 or above being at risk, 15 -19 high risk, 20 and above very high risk [21]. A newly developed ulcer was visualised on her posterior ankle to calf (Fig. 1.) most likely due to bandage slippage and the woman's inability to lift her leg to remove the pressure. Her dorsalis pedis pulse felt weak but palpable, the arterial duplex results confirmed no occlusion in tri vessel arterial supply. Her nutrition was suboptimal and this consisted of a high carbohydrate, fat and sugar diet. The woman reported poor hydration due to her limited mobility, commenting that she avoided drinking because she couldn't get to the toilet and was not having regular bowel motions.

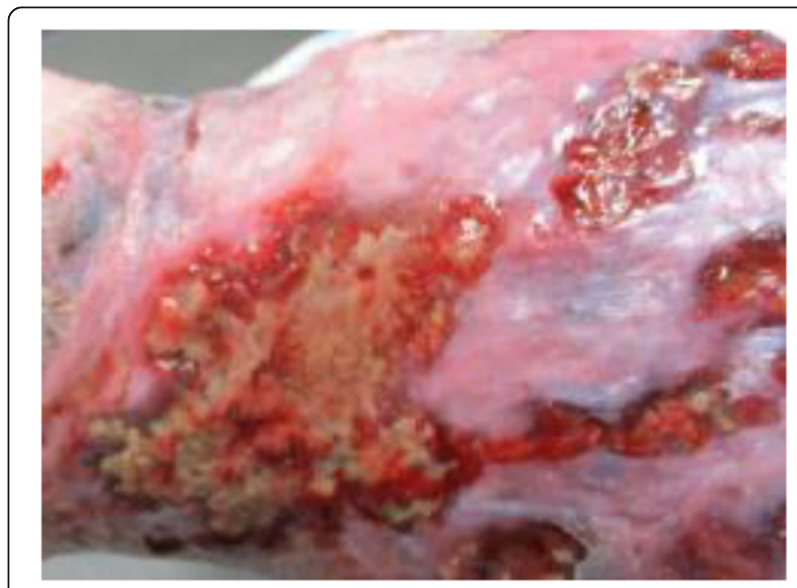

Fig. 1 Tissue damage from bandage slippage posterior lower calf 


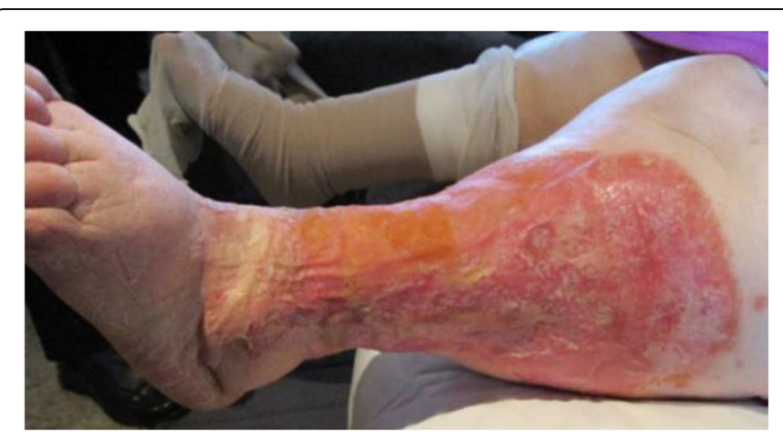

Fig. 2 Pre IPC therapy: Circumferential ulceration and tissue maceration from continued oedema and large exudate leakage

\section{Interventions}

The intervention commenced following the Wound Nurse Specialist review in August of 2015 and multidisciplinary case review at the woman's home. Involved in the review were the woman her carer' the GP, Occupational Therapist, Physiotherapist, Dietician, Community Nurse and Wound Nurse Specialist where a management plan was developed promoting the woman's involvement in her own wound care planning. The overall treatment was aimed at, reducing the oedema, preventing further pressure injuries, improving skin hygiene and nutritional support to achieve the woman's goal of wound healing, improving mobility and increasing independence and social activity.

A bariatric hospital bed was arranged by the Occupational Therapist, enabling the woman to elevate her legs to promote venous return and reduce staff risk of injury during dressing changes. A shower chair was provided enabling the woman to attend showers to improve personal hygiene and her feelings of self-worth. Nutrition support was also provided to optimise her nutrition for wound healing whilst monitoring weight loss was guided by the dietician who worked with both the woman and her carer providing education and support for meal planning. The physiotherapist attended the woman's home weekly to provide lymphatic drainage massage and exercise physiology including mobility training.

Medications were reviewed by a cardiologist who commenced her on oral furosemide 40mg twice per day. Safety clearance was provided by both a vascular surgeon and cardiologist to use IPC. IPC was commenced using bilateral thigh length leg garments at $40 \mathrm{mmHg}$ pressure, 2 cycles per minute, 40 -60 minute session duration attended three times per day. The woman was encouraged to increase frequency of sessions when she was able. The IPC therapy was attended at home by the woman and her carer' following Initial session with the Wound Nurse Specialist to educate the woman and her carer' on IPC use and pre-therapy breathing exercise. The woman continued leg elevation and calf pump exercises three times per day and commenced daily mobility with a four wheeled walker in conjunction with IPC.

Dressings were attended in the home second daily by community nurses for the first two weeks included in regime was leg hygiene, betadine wash, and $\mathrm{PH}$ neutral cream to surrounding tissue, Inadine (pharmaceutical topical antimicrobial) was applied to raw areas with an absorbent padding, tubi-fast to secure and tubi-grip. Home nursing visits were reduced to three times per week for weeks three to four, during this time community nurses continued to educate the woman and her carer to self - manage dressings. From week five to six frequency of home nursing visits were reduced to twice per week. Home nursing visits were reduced to weekly for the following six weeks. Fortnightly community nursing home visits to monitor and support self - management continued until the woman was discharged from community wound service in March of 2016.

\section{Follow-up and outcome}

Twelve weeks following the introduction of IPC therapy, this woman accomplished a significant weight loss of $36 \mathrm{~kg}$, lowering her BMI from 62.83 to 47.65 . There was also a reduction of over $10 \mathrm{~cm}$ in thigh circumference, her right thigh measured at $79 \mathrm{~cm}$ and left thigh measured at $82 \mathrm{~cm}$. There was substantial wound healing achieved (complete epithelialisation with no exudate leakage or skin irritation) (Fig. 3) and wound pain scale score reduced to $0 / 10$. There was noted improvement in skin condition and softening of sub-dermal tissue. The skin folds to her legs had reduced (Figs. 4 \& 5) and this woman reported improved personal hygiene and selfesteem, she now enjoyed socialising outside the home.

Concordance with IPC therapy during this time was self - reported with the woman stating "only an occasional session was missed". This woman was now able to bend her knees and attend heel lifts and continued daily mobilisation - walking around the outside of her house to the clothes line and back independently with a four wheeled walker. Her independence with activities of daily living was improved, the woman reported feeling proud of herself as she was managing to shower, dry and dress without

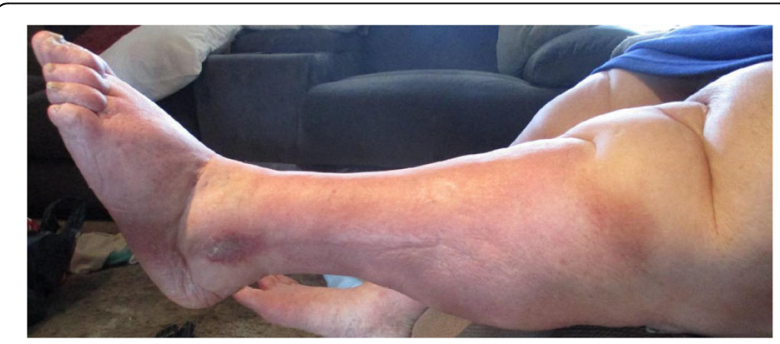

Fig. 3 Week 6: post IPC therapy initiation; no exudate, complete epithelialisation, reduced oedema 


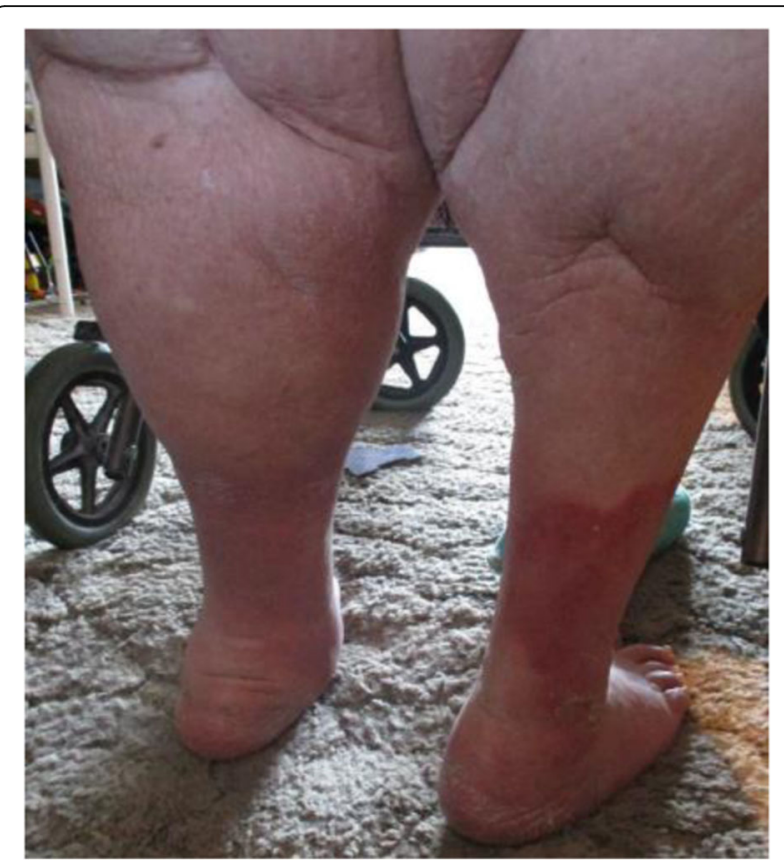

Fig. 4 Week 10: client mobilising, reduced oedema fibrotic tissue softening

assistance and reported being able to attend housework such as "cleaning out kitchen cupboards" which she hadn't attended to in years her urinary incontinence resolved and bowel motions were regular.

This woman reported some initial discomfort with an increase in frequency of urination when first commenced on IPC, stating this settled within two weeks. Her Waterlow assessment was updated and scored 10, the reduced risk score predominantly resulting from

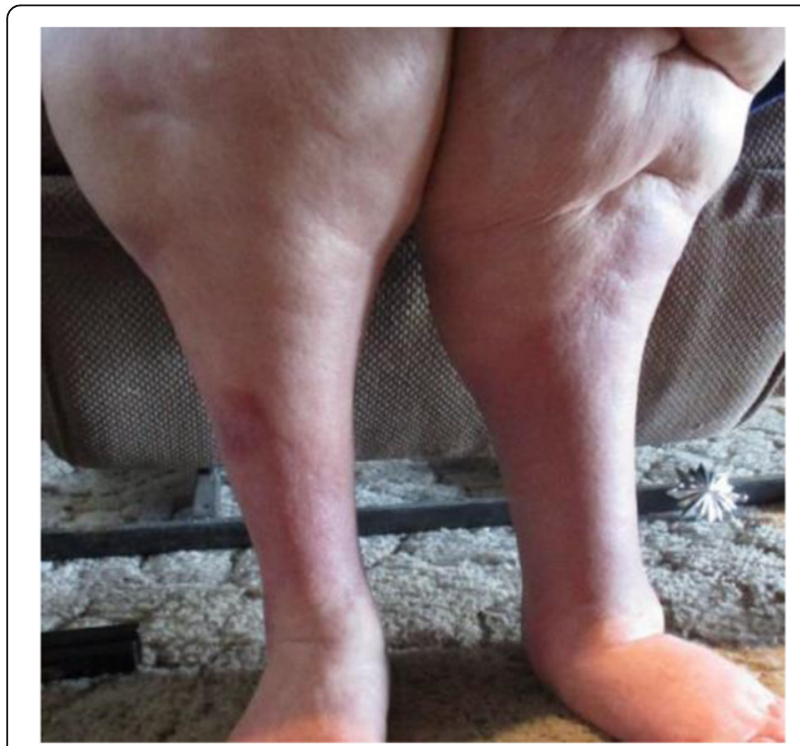

Fig. 5 Week 12: no ulcerations or exudate leakage improved mobility and continence. There were no reported episodes of infections during or after the twelve weeks IPC therapy. A follow up phone call was attended in April of 2016 where the woman confirmed her continued self-management with IPC and healthy life choices to prevent ulcer recurrence. The woman reported no further ulcers or infections had developed.

\section{Patient perspective of using IPC at home twelve weeks on}

When asked how she felt about her experience with her chronic wounds, before and after IPC she responded;

"I felt very depressed before because my legs kept leaking, they smelt bad and hurt a lot, I didn't want to leave the house, soon I just wasn't able to, and I couldn't even walk to the toilet or wash myself. I found the IPC pump easy to use, at first my daughter had to put it on, now I can do it myself. My wounds are healed and I'm walking more every day, I can get to the toilet so don't have accidents anymore. I have gone out with friends I haven't seen for ages and can now play with my grandchildren, I feel much happier now"

\section{Discussion}

It is widely accepted that CLU development is primarily attributed to CVI with Gold standard treatment including compression bandaging [13], in combination with other measures such as physiotherapy, diet, patient education, pressure management and antibiotics where required $[1,8,21]$. Developing treatment methods such as transcutaneous electrical nerve stimulation (TENS) has shown some improvement, with VLU healing in people with multiple co-morbidities [22] but further investigation of this method is required to improve level of evidence. TENS is not part of current Australian guideline recommendations (1) as such public access to this treatment is not available to clients of the service.

The practice of bandaging however is not always practical in clinical practice with a variety of barriers being identified as preventing effectiveness of static compression therapy (bandaging) $[15,19]$. This case report outlined barriers this woman faced with traditional compression, the introduction of IPC enabled her access to treatment in the home. A good result was achieved in this individual case. The woman improved her quality of life, with complete resolution of wounds, improved mobility her pain resolved and independence increased. The costings of consumables and nursing hours in this instance were collected eight weeks pre and twelve weeks post IPC therapy showing a saving of approx. $\$ 250$ per week for the community health service.

Because other treatment measures were used in combination with IPC in this instance and concordance was selfreported it is difficult to measure the significance IPC alone 
had on this woman's wound healing outcomes. A review by Nelson et al. [12] suggests further trials are required to determine reliability of current evidence on IPC use for leg ulcer healing. This is the first documented study using IPC in the home for treatment of CLU. Results from this case, support the need to further explore the use of IPC as an adjunct therapy for leg ulcer management in the community setting. A full clinical trial of the use of IPC at home is in the planning stages.

\section{Abbreviations}

CLU: Chronic leg ulcer; CVI: Chronic venous insufficiency; IPC: Intermittent pneumatic compression; VLU: Venous leg ulcer

\section{Acknowledgements}

Acknowledgement section woman/client for allowing us to share her story, Nepean Blue Mountains Local Health District (NBMLHD), Clinical Nursing Research and Practice Department (CNRPD).

\section{Funding}

This project was not funded.

\section{Availability of data and materials}

Consent for publication of raw photos and data obtained from study participant.

\section{Authors contributions}

KY supported the case client in this case report and reported the findings. $\mathrm{KY}$ and HNC was involved in the literature review. KY, HNC and LW were involved in the editing and proofing of draft manuscript of this case report. All authors read and approved the final manuscript.

\section{Ethics approval and consent to participate}

Ethics approval was granted for the Clinical Case Report and Media and Image use from the Nepean Blue Mountains Local Health District Human Research Ethics Office. The client also provided informed consent to participate.

\section{Consent for publication}

A media and image consent form along detailing the consent to publish was granted on the 10/05/16 by the patient.

\section{Competing interests}

The authors declare no competing interests.

\section{Publisher's Note}

Springer Nature remains neutral with regard to jurisdictional claims in published maps and institutional affiliations.

\section{Author details}

${ }^{1}$ Nepean Hospital, Nepean Blue Mountains Local Health District, Derby Street, Kingswood, NSW 2747, Australia. ${ }^{2}$ Western Sydney University/Nepean Blue Mountains Local Health District, Sydney, Australia. ${ }^{3}$ Centre for Nursing Research and Practice Development, Nepean Hospital, First Floor - Court Building - Nepean Hospital, PO Box 63, Penrith, NSW 2751, Australia. ${ }^{4}$ School of Nursing and Midwifery, Western Sydney University/Nepean Blue Mountains Local Health District, Sydney, Australia.

Received: 21 March 2017 Accepted: 5 September 2017

Published online: 21 September 2017

\section{References}

1. Australian Wound Management Association. Australian And New Zealand Clinical Practice Guideline For The Prevention And Management of Venous Leg Ulcers. Barton, ACT: AWMA; 2011.

2. McGuiness B. A Guide for talking to politicians regarding wound care. Australia: Victoria; 2012.
3. Franks PJ, Barker J, Collier M, Gethin G, Haesler E, Jawien A, Laeuchli S, Mosti $\mathrm{G}$, Probst S, Weller C. Management of patients with venous leg ulcers: challenges and current best practice. J Wound Care. 2016;25 (sup6):S1-67.

4. Agale SV. Chronic leg ulcers: epidemiology, aetiopathogenesis, and management. Ulcers; 2013. p. 2013.

5. Graves N, Zheng $\mathrm{H}$. Modelling the direct health care costs of chronic wounds in Australia. Wound Pract \& Res: J Aust Wound Manag Assoc. 2014; 22(1):20.

6. Kapp S, Santamaria N. Chronic wounds should be one of Australia's National Health Priority Areas. Aust Health Rev. 2015;39(5):600-2.

7. Smith E, McGuiness W. Managing venous leg ulcers in the community: personal financial cost to sufferers. Wound Pract \& Res: J Aust Wound Manag Assoc. 2010;18(3):134

8. Harding K, Dowsett C, Fias L, Jelnes R, Mosti G, Öien R. Simplifying venous leg ulcer management: consensus recommendations. Wounds Int. 2015.

9. Wounds International. International Best Practice: Optimising patient involvement in wound management. London: Wounds Int Schofield Healthc Media. 2016

10. Comerota AJ. Intermittent pneumatic compression: Physiologic and clinical basis to improve management of venous leg ulcers. J Vasc Surg. 2011;53(4): 1121-9.

11. Reyes APD, Partsch H, Mosti G, Obi A, Lurie F. Report from the 2013 meeting of the International Compression Club on advances and challenges of compression therapy. J Vasc Surg: Venous Lymphat Disord. 2014;2(4):469-76.

12. Nelson EA, Hillman A, Thomas K. Intermittent pneumatic compression for treating venous leg ulcers. The Cochrane Library. 2014.

13. Kordzadeh A, Jonas A, Panayiotopoulos YP. Intermittent Pneumatic Compression in Treatment of Chronic Venous Leg Ulcers: A Case Report and Review of Literature. Case Rep Clin Med. 2014;2014

14. European Wound Management Association. Position document: hard-toheal wounds: a holistic approach. London: MEP Ltd; 2008.

15. Tandler SF. Challenges faced by healthcare professionals in the provision of compression hosiery to enhance compliance in the prevention of venous leg ulceration. EWMA Journal. 2016;16(1).

16. Partsch $\mathrm{H}$. Intermittent pneumatic compression in immobile patients. Int Wound J. 2008:5(3):389-97.

17. Nelson EA, Mani R, Thomas K, Vowden K. Intermittent pneumatic compression for treating venous leg ulcers. Cochrane Libr. 2011.

18. Van Hecke A, Grypdonck M, Defloor T. A review of why patients with leg ulcers do not adhere to treatment. J Clin Nurs. 2009:18(3):337-49. doi:10.1111/j.1365-2702.2008.02575.x.

19. Van Hecke A, Grypdonck M, Beele H, Vanderwee K, Defloor T. Adherence to leg ulcer lifestyle advice: qualitative and quantitative outcomes associated with a nurse-led intervention. J Clin Nurs. 2011;20(3-4):429-43. doi:10.1111/j.1365-2702.2010.03546.x.

20. Care Group. CARE Checklist 2013. CARE case Report Guidelines. 2013. http:// www.care-statement.org/. Accessed 23 Feb 2017.

21. Australian Wound Management Association. Pan Pacific clinical practice guideline for the prevention and management of pressure injury. Osborne Park: Cambridge Media: Aust wound Manag Assoc. 2012.

22. Ingves MV, Power AH. Two Cases of Transcutaneous Electrical Nerve Stimulation of the Common Peroneal Nerve Successfully Treating Refractory, Multifactorial Leg Edema. J Invest Med High Impact Case Rep. 2014;2(4) doi:10.1177/2324709614559839.

\section{Submit your next manuscript to BioMed Central} and we will help you at every step:

- We accept pre-submission inquiries

- Our selector tool helps you to find the most relevant journal

- We provide round the clock customer support

- Convenient online submission

- Thorough peer review

- Inclusion in PubMed and all major indexing services

- Maximum visibility for your research

Submit your manuscript at www.biomedcentral.com/submit
Biomed Central 